\title{
PERCEPTRON ANN CONTROL OF ARRAY SENSORS AND TRANSMITTERS WITH DIFFERENT ACTIVATION FUNCTIONS FOR 5G WIRELESS SYSTEMS
}

\author{
K.S. Senthilkumar ${ }^{1}$, K. Pirapaharan ${ }^{2}$, Norhuzaimin Julai ${ }^{3}$, P.R.P Hoole ${ }^{3}$, Al-Hj Othman ${ }^{3}$, R. Harikrishnan ${ }^{4}$, S.R.H. \\ Hoole $^{5}$ \\ ${ }^{1}$ Department of Computers and Technology, St. George's University, Grenada, WI \\ ${ }^{2}$ Department of Electrical and Communications Engineering, Papua New Guinea University of Technology, Lae, PNG \\ ${ }^{3}$ Department of Electrical and Electronic Engineering, Universiti Malaysia Sarawak, Sarawak, Malaysia \\ ${ }^{4}$ Department of Electrical Engineering, University of Malaya, Malaysia \\ ${ }^{5}$ Department of Electrical and Computer Engineering, Michigan State University, Michigan, USA
}

(Correspondence: ksskumar16@gmail.com)

\begin{abstract}
Successful development of a fast, low memory Perceptron Artificial Neural Network (ANN) based electromagnetic signal processor for array sensors has spurred the interest in embedding the electromagnetic signal processor in highly portable (e.g. to be attached to a drone) Arduino as well as in fast parallel Graphic processing Unit (GPU) based systems. In this paper we report the initial results on the embedded system, while also improving on the perceptron based array beam forming with effective activation functions. The paper also explores the effectiveness of different geometrical arrangements of the array system for different applications from wireless communication, medical EEG data acquisition and analysis to power cable condition monitoring and diagnosis.
\end{abstract}

Keywords-Complex activation function, Perceptron, inteligent array sensors, , Smart Antenna, Adaptive Array, 5G physical layer, Artificial Neural Network Antenna.

\section{INTRODUCTION}

The new generation of wireless systems, namely the $5 \mathrm{G}$ Systems, will heavily depend on the efficient design and deployment of smart antennas which will form the backbone for making the mobile stations not only able to transmit, receive and process their own specific communication and control signals, but also that of other users as the gradual move will be to transfer the functions of the base station in the present wireless systems onto the mobile station. The Space Division Multiple Access (SDMA) capability of multibeam, steerable smart antennas help to eliminate to a significant extent the interference signals by using change in air interface capabilities of smart antennas. Co-channel interference is reduced by smart antennas by allowing different channels to use the different beams generated by the smart antenna. The search is to develop smart antennas that provide efficient performance in relation to cost, complexity of the antenna structure and computational burden and directional gain $[1,2]$. This will need to be done while forming an optimum desired beam and minimum power demand from the battery $[3,4,5]$. This is the focus point of the new Perceptron ANN based smart antenna which is shown to allow for versatile control of desired beam while keep computational power and memory to the minimum [6]. Although traditionally neural network architectures operate on real-valued inputs, signals in array antennas are complex inputs. Training the neural networks using complex inputs was done using techniques like the back-propagation, Hopfield model and perceptron learning rules.

Recently, interests on ANN have moved towards the usage of complex-valued neural networks on applications in imaging, remote sensing, and realistic mobile systems, such as Global System for Mobile communications (GSM), 5G wireless systems and artificial neural information processing.. Widrow, Mc Cool and Ball [7] proposed a complex valued neural network algorithm in 1975 by making use of a complex least mean squares (LMS) algorithm. Following this, for signal processing applications a learning algorithm based on complex values was reported by Kim and Guest [8]. Further work using other complex activation functions was reported by Georgiou and Koutsougeras [9]. Deville [10] implemented a complex activation function for digital Very Large Scale Integration (VLSI) neural networks and claimed that it required less hardware than the conventional real valued neural network. Another complex-valued activation function was implemented by Deville for digital Very Large Scale Integration (VLSI) neural networks to prove that it required less hardware than the conventional real-valued neural network [10].

This paper explores the best function to use for the ANN activation function to achieve desired beam maximization and interference minimization while seeking fast and accurate antenna training and minimizing of repeated calculations, which will be a drain on the battery, when rotating the main beam to maintain communication with a moving mobile station. The consideration of the speed of the mobile station 